\title{
Color Characteristics in RBG Space under Outdoor Illumination in Tropical Region
}

\author{
Anup Vibhute \\ Assistant Professor, \\ BMIT, Solapur (India)
}

\author{
S K Bodhe \\ $\mathrm{PhD}$, Professor, COEP \\ Director, Bosh Technology, Pune
}

\begin{abstract}
Color is an important parameter in object recognition for human being and machine vision. The color constancy models developed by researchers always tend to mapping process. This paper studies the behavior of the RGB colors in outdoor illumination in tropical region. The colors values changes in the morning and evening illumination conditions and Euclidean distance calculated between planes shows the same trend for all planes.
\end{abstract}

\section{Keywords - \\ Color constancy, illumination, luminance, RGB space}

\section{INTRODUCTION}

The term color can be defined as attribute of human vision perception with any combination of chromatic and achromatic contents. Chromatic contents are colors viz. red, green, blue and its combinations. Achromatic contents are gray color between black and white. Image is a function of illumination and reflection where as color image is a function of illumination, reflection and sensor sensitivity. $[1,2]$

Illuminating Engineering Society of North America defines light as a radiant energy that excites retina and produce a visual sensation. Reflection are classified into three categories- Specular, Spread and diffused which occurs on polished, rough and matte surfaces respectively. The concepts of illumination and reflectance along with the different laws viz. inverse square law, Lambert's cosine law were discussed. [6]

Colorimetrical RGB encoding standards are generally used for color reproduction and color information exchanges. CIE standards are measured under D65 illuminant. When image is viewed observer see light from image and viewing conditions depends upon adapted white point, surround, luminance level and viewing flare. So improper encoding schemes can severely damage color conversions .Illuminants affects the device color-space characteristics and transformation. [2]

Color constancy is the phenomenon or ability of human to correct color deviation caused by different illumination. Color. constancy for machine vision always remained a challenge for researchers. Many researchers have presented the models for the color constancy. Conscious and unconscious inferences regarding color constancy discussed along with solution and application to color complexity in color constancy. [3]

Putting the limitations of CIE illuminants, the models were discussed for illumination and surface reflectance in outdoor conditions. The model also predicts the color in outdoor conditions with various case studies of surfaces. Models viz. CIE daylight, Daylight indexed by context, Normalized reflectance model, etc. [4] Different color sets were indexed under four household illuminations. It was observed that the color contents changes dramatically resulting in poor index and it was improved by removing illumination biasing with color mapping. [5]

An algorithm for radiometric calibration under illumination change has been proposed for outdoor scenes. Sinusoidal model proposed for computing exposure which represents the daily motion of the sun. Camera response function calculated on the basis of changing illumination. [7] Outdoor color discriminant classifier based on adaptive similarity based classifier was proposed which gives greater accuracy than the conventional classifiers.[10] Other white balancing techniques were discussed by many researchers where the source of light is standard like D65 were used. [8,9]

Color complexity with illumination motivated us to study the behavior of the RGB colors. Experiments carried out under controlled environment may result into models for color constancy for specific application. This paper evaluates RGB patterns in presence of outdoor illumination.

\section{METHOD AND MATERIALS}

\subsection{Image Acquisition:}

In this experiment color characteristics in RGB space under outdoor illumination studied in tropical region in India. The experiment was carried out on sunny day in the winter with three different colored cloths- Red, Green, and Blue. Images were taken from $7 \mathrm{am}$ to $6 \mathrm{pm}$ at an interval of a hour. The luminance was measured with Luxmeter Lutron LX-1108 which is characterized by photodiode as sensor, color correction filter and spectra meet CIE photopic. Images captured with digital camera Kodak C183 having CCD sensor at the same position. 
Table 1: RGB Euclidean distance of each plane for Red, Green and Blue Cloth.

\begin{tabular}{|r|r|r|r|r|r|r|r|r|r|r|}
\hline \multirow{2}{*}{ TIME } & \multirow{3}{*}{ Max } & \multicolumn{3}{|c|}{ Red cloth } & \multicolumn{3}{|c|}{ Green Cloth } & \multicolumn{3}{|c|}{ Blue cloth } \\
\cline { 3 - 11 } & \multicolumn{1}{|c|}{ RM_ED } & GM_ED & BM_ED & RM_ED & GM_ED & BM_ED & RM_ED & GM_ED & BM_ED \\
\hline $7: 00$ & 1311 & 223 & 190 & 139 & 157 & 158 & 99 & 217 & 135 & 190 \\
\hline $8: 00$ & 23200 & 261 & 200 & 172 & 104 & 136 & 90 & 94 & 58 & 85 \\
\hline $9: 00$ & 59500 & 249 & 195 & 162 & 104 & 133 & 86 & 107 & 66 & 96 \\
\hline $10: 00$ & 78300 & 244 & 193 & 158 & 110 & 138 & 89 & 120 & 74 & 107 \\
\hline $11: 00$ & 125700 & 236 & 185 & 153 & 106 & 134 & 86 & 121 & 79 & 95 \\
\hline $12: 00$ & 114900 & 232 & 179 & 152 & 104 & 131 & 85 & 112 & 69 & 100 \\
\hline $13: 00$ & 89500 & 232 & 178 & 153 & 104 & 131 & 85 & 110 & 68 & 99 \\
\hline $14: 00$ & 99200 & 241 & 187 & 157 & 102 & 130 & 84 & 103 & 64 & 93 \\
\hline $15: 00$ & 53100 & 238 & 185 & 156 & 97 & 126 & 83 & 102 & 63 & 92 \\
\hline $16: 00$ & 42800 & 247 & 190 & 163 & 91 & 122 & 82 & 95 & 59 & 86 \\
\hline $17: 00$ & 13820 & 241 & 186 & 158 & 92 & 119 & 78 & 97 & 60 & 89 \\
\hline $18: 00$ & 159.2 & 255 & 206 & 163 & 124 & 142 & 88 & 219 & 136 & 193 \\
\hline
\end{tabular}

Two assumptions are made that each pixel value is independent of neighboring pixel and cloth is illuminated by Sun as source and other reflections are not considered as the images were taken under open sky.

\subsection{Image Analysis:}

RGB space utilized as it is plays vital role in colorimetry and CIE color matching functions. Many applications viz. pictures, film printing, television, etc. use RGB primary.[2]

Mathematically a color image is represented as,

$$
E_{k}(x, y, \lambda)=\int R(x, y, \lambda) L(\lambda) S_{k}(\lambda) d \lambda
$$

a product of three variables, namely, $\mathrm{R}(\mathrm{x}, \mathrm{y}, \lambda)$ the surface reflectance, $L(\lambda)$ the illumination property, and $S_{k}(\lambda)$ the sensor characteristics, as a function of the wavelength $\lambda$, over the visible spectrum. As the Material and image device are unchanged, color will be the function of illumination.

RGB planes are separated and mean value of the plane was determined. The Euclidean distance between these planes for each color cloth was calculated.

$$
\begin{aligned}
& \text { R_E }=\sqrt{(R-G)^{2}+(R-B)^{2}} \\
& \text { G_E }=\sqrt{(G-R)^{2}+(G-B)^{2}} \\
& \text { B_E }=\sqrt{(B-R)^{2}+(B-G)^{2}}
\end{aligned}
$$

The values calculated are shown in table 1. The Histograms and graphs plotted with respect to time are shown in figure 1 to 3 .
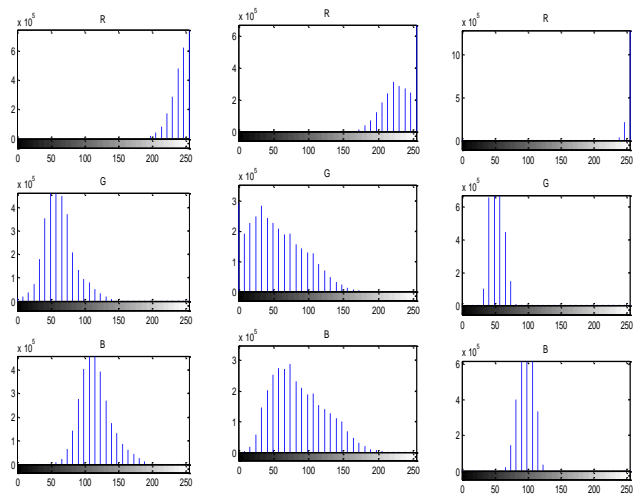

Fig 1a: Histogram for Red cloth at 7am, 11am \& 6 pm 


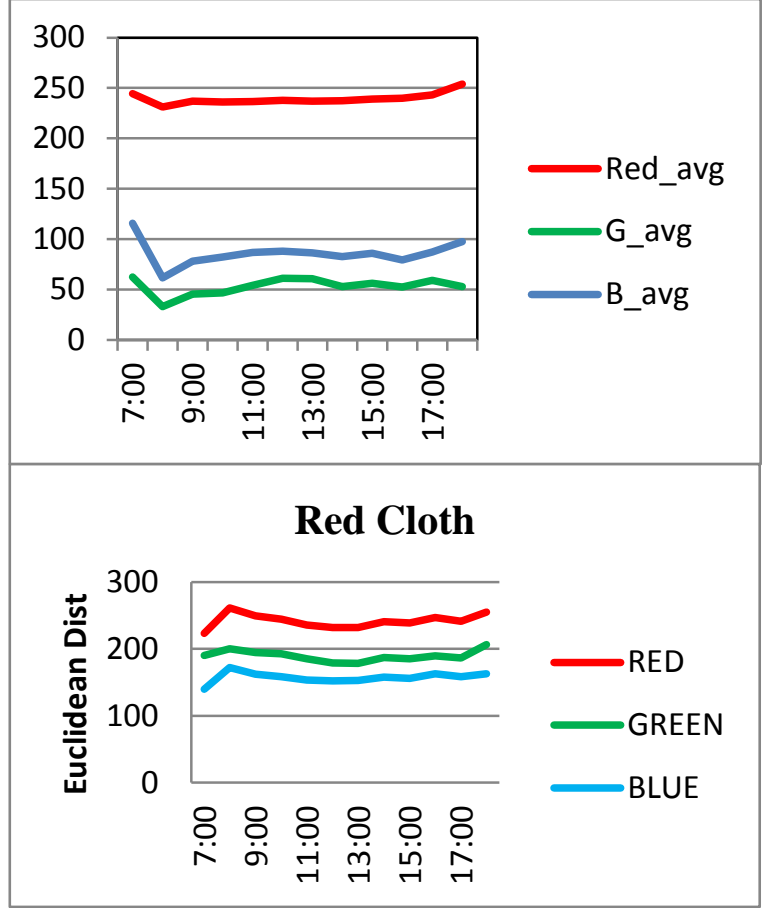

Fig 1b: RGB mean values and Euclidean distance for red cloth
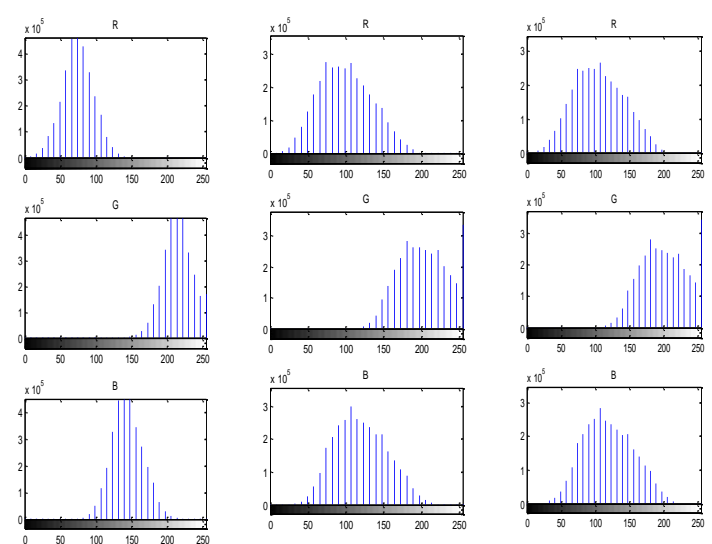

Fig 2a: Histogram for Green cloth at 7am, 11am \& $6 \mathrm{pm}$

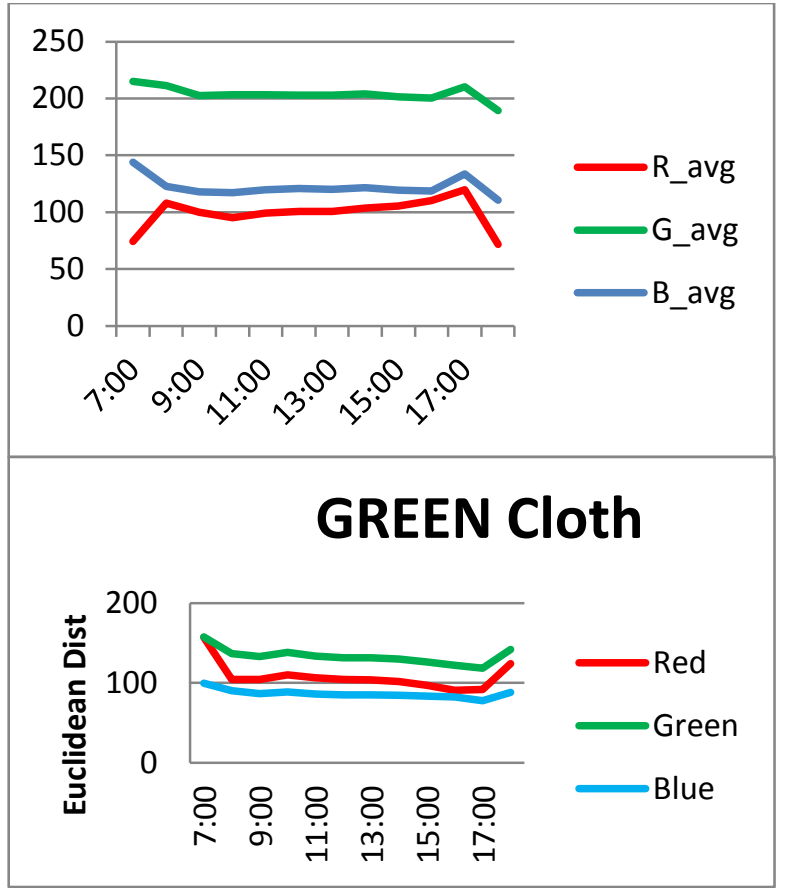

Fig 2b: RGB mean values and Euclidean distance for Green cloth


Fig 3a: Histogram for Blue cloth at 7am, 11am \& 6pm 


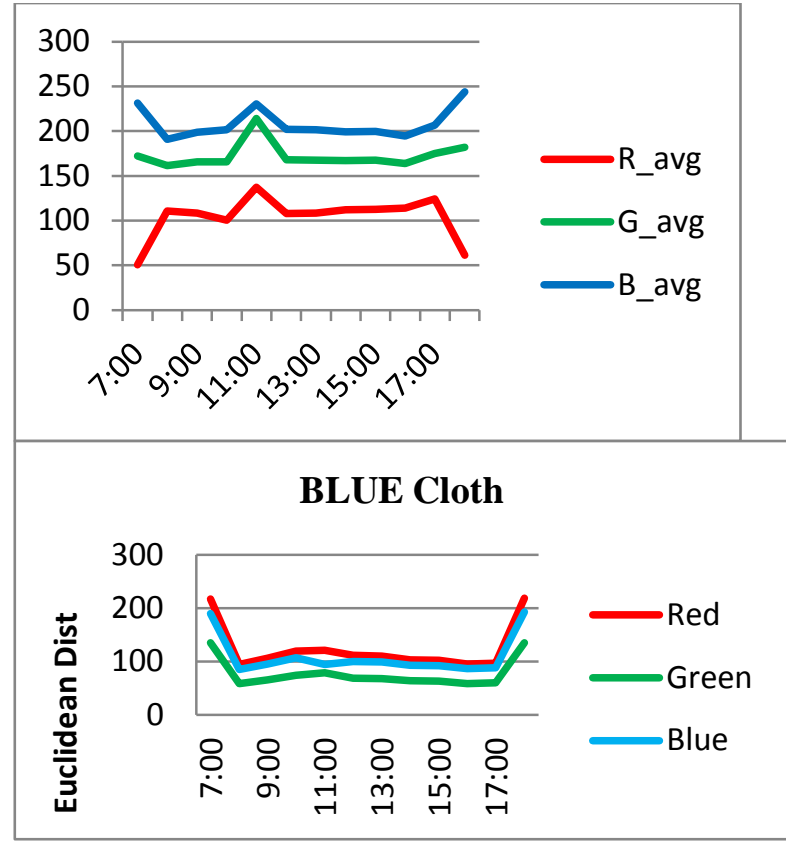

Fig 3b: RGB mean values and Euclidean distance for Blue cloth

\section{RESULT \& DISCUSSION}

In case of Red and Green cloths the average value of respective color plane is higher than the other planes. The Red plane value dominates Green and blue planes by large margin. Green and Blue shows darkness in Red cloth and can be seen from histograms. The Euclidean distance (ED) shows almost constant trend for all the planes in red cloth.

For green cloth $\mathrm{G}$ planes dominates other plane values. $\mathrm{R}$ and B plane shows low contrast. The Euclidean Distance follow same trend in case of green and blue planes but in morning and evening the Red ED changes observed is more.

For Blue cloth Blue and Green values follow same trend but red color shows the variations in morning and evening sessions, where as the ED shows the same trend between planes.

The values observed for Red, Green and Blue cloth can be stated as

$$
\begin{aligned}
& \text { Red }-R>G>B \\
& \text { Green }-G>B>R \\
& \text { Blue }-B>G>R
\end{aligned}
$$

\section{CONCLUSION}

Illumination plays very important role in color characteristics. Behavioral characteristics of different colors studied under this experiment shows that the values of red, green and blue plane in morning and evening shows changes when illumination is less and almost remain constant in late morning and afternoon sessions. The ED almost remains constant for all the colors and follows same trends.

Lambert's cosine law can be used to enhance the results. These results can be used to calibrate cameras with change in illumination [7]. Statistical approach to RGB data may be used for further study. The results of the experiment can be extended to map the colors in the morning and evening sessions for color constancy. The plane values at the different illumination can be extended for color segmentation in RGB.

\section{REFERENCES:}

[1] Gonzalez Rafael C., Richard E woods, "Digital Image Processing", $2^{\text {nd }}$ Edition.

[2] Henry R. Kang, 2006, "Computational Color Technology", SPIE.

[3] David Hilbert, "Color constancy and complexity of color".

[4] Shashi Buluswar and Bruce Draper, 2001, "Color models for outdoor machine", Computer vision and image understanding, volume 84, 1-29.

[5] Graham D. Finlayson and Gui Yun Tian, 1999, "Color indexing across illumination", challenge of image retrieval, pp.1-7.

[6] Alma E. F. Taylor, 2000," Illumination Fundamentals", Lighting Research Center, pp 1-48.

[7] Seon Joo Kim, Jan-Michael Frahm and Marc Pollefeys, 2008, "Radiometric Calibration with Illumination Change for Outdoor Scene Analysis", CVPR08, pp 1-8.

[8] Varsha Chikane and Chiou-shann Fuh,2006," Automatic white balance for digital still cameras," Jpurnal of information science and engineering, vol. 22., pp. 497 509 .

[9] Ching-Chih Weng, Homer Chen, and Chiou-Shann Fuh," A novel automatic white balance method for digital still cameras", Taiwan,vol.12, no.4, 2006, pp. 4-9.

[10] O.Sojodishijani, A.R. Ramli, V. Rostami,K. Samsudin,M.I. Saripan," Just in time outdoor color discrimination using adaptive similarity based classifier",IEICE Electronic Express, Vol 7, No.5, pp 339-345,2010. 